\title{
Searches for lepton flavour violation and baryon number violation at $\mathrm{LHCb}$
}

\author{
Jon Harrison \\ on behalf of the LHCb Collaboration
}

12th International Workshop on Tau Lepton Physics

Nagoya, Japan, 17th-21st September, 2012

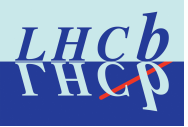

$$
\underset{1824}{\text { MANCHESTER }}
$$




\section{$\tau^{-} \rightarrow \mu^{-} \mu^{-} \mu^{+}$at LHCb}

- $\tau^{-} \rightarrow \mu^{-} \mu^{-} \mu^{+}$possible in SM

+ neutrino oscillations, but highly suppressed

$\rightarrow \mathcal{B} \ll 10^{-40}$

- Rates enhanced by various new physics scenarios e.g. MSSM + massive neutrinos, RPV SUSY, Littlest Higgs with $\mathcal{T}$-Parity

- Predicted branching fractions for some models close to current experimental limits for some regions of parameter spaces
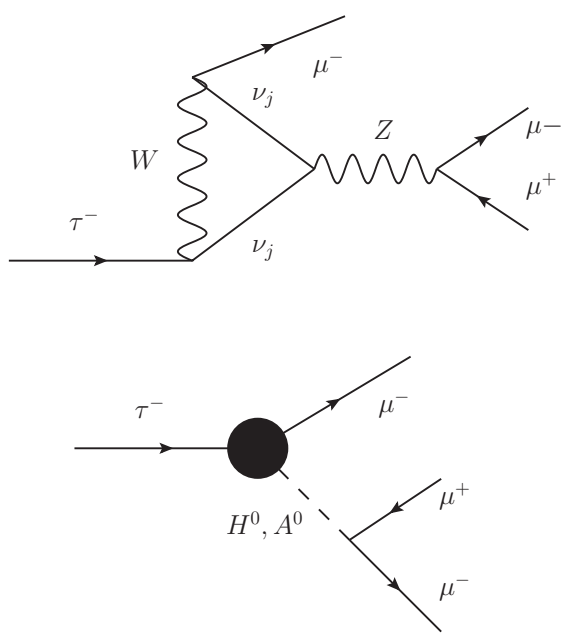


\section{Current status}

- $\tau^{-} \rightarrow \mu^{-} \mu^{-} \mu^{+}$yet to be observed

- Current experimental limits (at 90\% C.L.):

\begin{tabular}{|c|c|c|}
\hline Experiment & Upper limit & Number of $\tau^{+} \tau^{-}$pairs \\
\hline \hline BaBar $^{1}$ & $3.3 \times 10^{-8}$ & $430 \times 10^{6}$ \\
\hline Belle $^{2}$ & $2.1 \times 10^{-8}$ & $719 \times 10^{6}$ \\
\hline
\end{tabular}

- But can also set limits at a hadron collider... (See Diego's talk at 4pm tomorrow for more information on the $\mathrm{LHCb}$ detector)

${ }^{1}$ Phys. Rev. D 81, 111101 (2010)

${ }^{2}$ Phys. Lett. B 687, 139-143 (2010) 


\section{Analysis strategy}

- Inclusive $\tau$ cross section is $79.5 \pm 8.3 \mu \mathrm{b}$ at $\sqrt{s}=7 \mathrm{TeV}$ in LHCb acceptance

- $1.0 \mathrm{fb}^{-1}$ collected in 2011 $\rightarrow 8 \times 10^{10} \tau$ produced

- $\tau$ mainly from $D_{s}^{-} \rightarrow \tau^{-} \nu_{\bar{\tau}}$ decays $(78 \%)$

LHCb Integrated Luminosity at 3.5 TeV in 2011

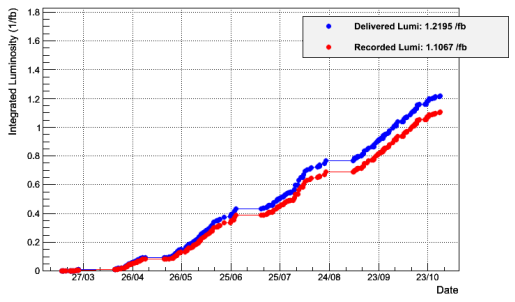

- Relative normalisation to $D_{s}^{-} \rightarrow \phi\left(\mu^{+} \mu^{-}\right) \pi^{-}$

- Loose cut-based selection followed by 3D classification:

(1) Decay topology and kinematics

(2) Particle identification

(3) Invariant mass

- Classifiers trained on simulated signal and calibrated on control channels

- Limit from $\mathrm{CL}_{s}$ method (J. Phys. G: Nucl. Part. Phys. 28 2693) 


\section{$\tau$ production at $\mathrm{LHCb}$}

- Relative contributions to $\tau$ production calculated using LHCb cross section measurements and LEP/B-factory branching fractions :

\begin{tabular}{|l|c|}
\hline Decay chain & Calc (\%) \\
\hline \hline$D_{s} \rightarrow \tau$ & $77.9 \pm 4.7$ \\
$D_{s} \rightarrow \tau$ & $67.8 \pm 4.8$ \\
$B_{X} \rightarrow D_{s} \rightarrow \tau$ & $10.1 \pm 2.1$ \\
$D^{-} \rightarrow \tau$ & $4.6 \pm 4.4$ \\
$D^{-} \rightarrow \tau$ & $4.4 \pm 4.2$ \\
$B_{x} \rightarrow D^{-} \rightarrow \tau$ & $0.3 \pm 0.3$ \\
$B_{x} \rightarrow \tau$ & $17.5 \pm 3.3$ \\
\hline
\end{tabular}

- Contributions from $Z, W$ and Drell-Yan are found to be negligible

- Correct MC to agree via reweighting 


\section{Event selection}

- Events required to pass LHCb trigger ( 45\% efficient)

- Then selected via:

\begin{tabular}{|c|c|}
\hline Cut & $\tau \rightarrow \mu \mu \mu \quad D_{S} \rightarrow \phi(\mu \mu) \pi$ \\
\hline$\mu^{ \pm}$and $\pi^{ \pm}$ & \\
\hline$p_{T}$ & $>300 \mathrm{MeV} / \mathrm{c}$ \\
\hline Track $\chi^{2} / N_{d o f}$ & $<4$ \\
\hline IP $\chi^{2} / N_{d o f}$ & $>9$ \\
\hline$\phi(1020)$ & \\
\hline$\Delta \mathrm{m}$ & $<20 \mathrm{MeV} / \mathrm{c}^{2}$ \\
\hline $\begin{array}{c}\tau^{ \pm} \text {and } D_{s}^{ \pm} \\
\Delta \mathrm{m}\end{array}$ & $<400 \mathrm{MeV} / \mathrm{c}^{2}$ \\
\hline Vertex $\chi^{2}$ & $<15$ \\
\hline $\mathrm{IP} \chi^{2}$ & $<225$ \\
\hline$C \tau$ & $>100 \mu \mathrm{m}$ \\
\hline Lifetime fit $\chi^{2}$ & $<225$ \\
\hline
\end{tabular}

- Also apply clone cut $\left(\mathrm{m}\left(\mu^{+} \mu^{+}\right)>250 \mathrm{MeV} / \mathrm{c}^{2}\right)$ and fiducial cuts

- Analysis performed blind with $\mathrm{m}(\tau) \pm 30 \mathrm{MeV} / \mathrm{c}^{2}$ excluded initially 


\section{$D_{s}^{-} \rightarrow \phi\left(\mu^{+} \mu^{-}\right) \pi^{-}$decays in data}

- $\mathcal{B}\left(D_{S} \rightarrow \phi(\mu \mu) \pi\right)$ from:

$$
\frac{\mathcal{B}\left(D_{s} \rightarrow \phi(K K) \pi\right)}{\mathcal{B}(\phi \rightarrow K K)} \mathcal{B}(\phi \rightarrow \mu \mu)=(1.33 \pm 0.12) \times 10^{-5}
$$

- Negligible contribution of non-resonant events in data $(<2 \%)$

- $N_{D_{s} \rightarrow \phi \pi}=45520 \pm 920$ from a gaussian fit to data

- $\phi(1020)$ reconstructed with a low background level

- Ingredients for normalisation factor (see later)

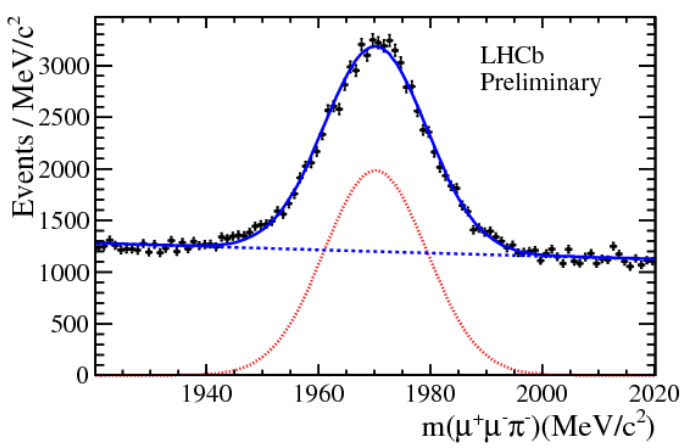




\section{Signal and background discrimination}

- Two multivariate classifiers, $\mathcal{M}_{3 \text { body }}$ and $\mathcal{M}_{\mathrm{PID}}$

- Use boosted decision trees with adaptive boosting

- $\mathcal{M}_{3 \text { body }}$ includes: vertex and track fit quality, vertex displacement, vertex pointing, vertex isolation and $\tau p_{T}$
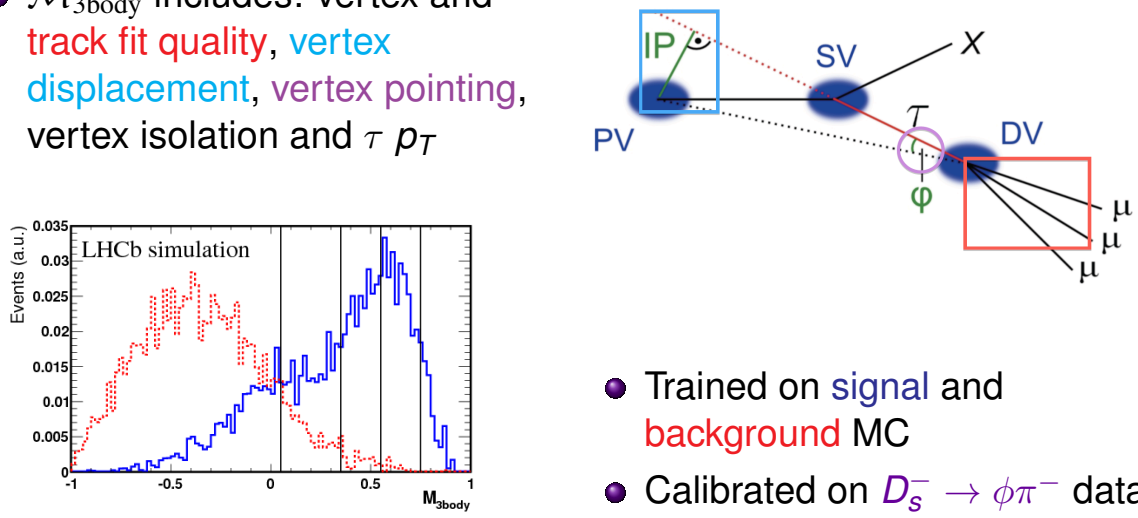

- Trained on signal and background MC

- Calibrated on $D_{s}^{-} \rightarrow \phi \pi^{-}$data 


\section{Signal and background discrimination $-\mathcal{M}_{\mathrm{PID}}$}

- $\mathcal{M}_{\mathrm{PID}}$ includes: information from RICH, ECAL, HCAL and muon chambers
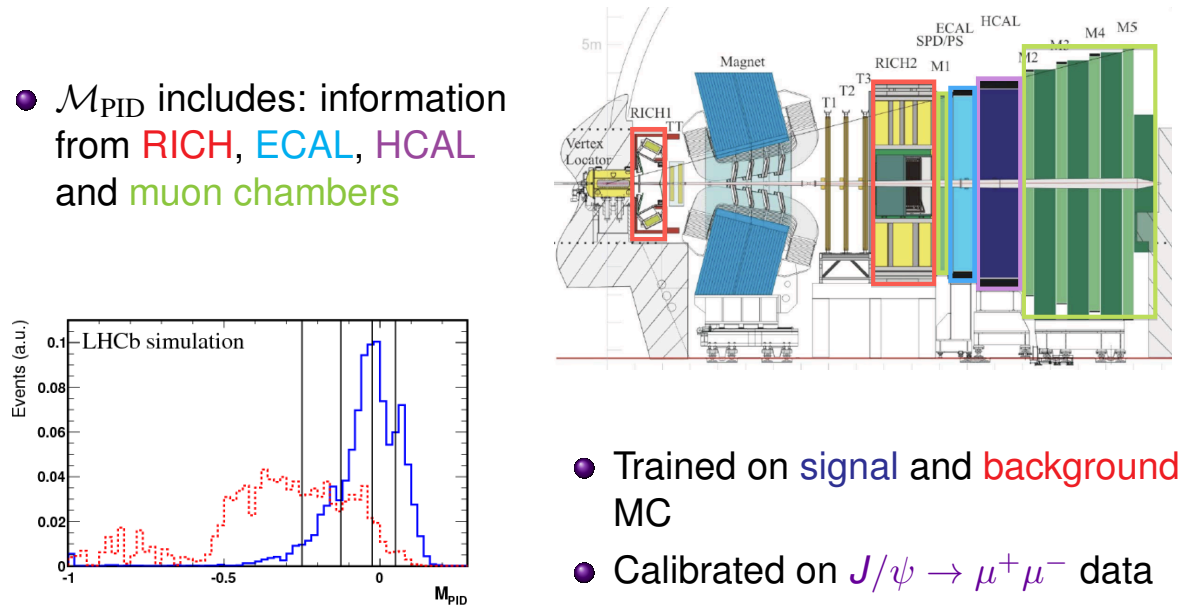

- Trained on signal and background MC

- Calibrated on $\mathrm{J} / \psi \rightarrow \mu^{+} \mu^{-}$data 


\section{Binning optimisation}

- We optimise both the number of bins and the position of the bin boundaries in the BDTs

- Use an iterative procedure to find the optimum of the figure of merit:

$$
\Delta L Q=2 \ln \left(Q_{S B}\right)-2 \ln \left(Q_{B}\right)
$$

where,

$$
\begin{aligned}
Q_{S B} & =\prod \frac{P\left(s_{i}+b_{i}, s_{i}+b_{i}\right)}{P\left(s_{i}+b_{i}, b_{i}\right)} \\
Q_{B} & =\prod \frac{P\left(b_{i}, s_{i}+b_{i}\right)}{P\left(b_{i}, b_{i}\right)}
\end{aligned}
$$

- 5 bins for both BDTs found to be optimal

- Detailed in arXiv:hep-ex/9706016 


\section{$\mu^{-} \mu^{-} \mu^{+}$mass binning}

- Signal mass window defined as $\mathrm{m}(\tau) \pm 15 \mathrm{MeV} / \mathrm{c}^{2}$

- Divided into 6 equally spaced bins

- Contains $\sim 90 \%$ of $\tau^{-} \rightarrow \mu^{-} \mu^{-} \mu^{+}$decays

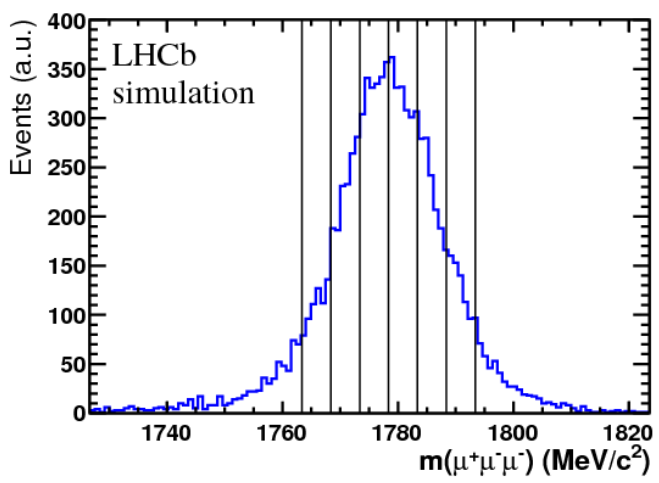

- Both central value of mass window and mass resolution corrected according to measured resolution and scaling at $\mathrm{LHCb}$

- Signal shape in mass window from fit to $D_{s}^{-} \rightarrow \phi \pi^{-}$events in data 


\section{Normalisation factor}

$$
\begin{aligned}
& \mathcal{B}(\tau \rightarrow \mu \mu \mu)= \\
& \mathcal{B}\left(D_{S} \rightarrow \phi(\mu \mu) \pi\right) \times \frac{f_{D_{S}}^{\tau}}{\mathcal{B}\left(D_{S} \rightarrow \tau \nu_{\tau}\right)} \times \frac{\epsilon_{D_{s} \rightarrow \phi \pi}}{\epsilon_{\tau \rightarrow \mu \mu \mu}} \times \frac{N_{\tau \rightarrow \mu \mu \mu}}{N_{D_{S} \rightarrow \phi \pi}}
\end{aligned}
$$

- $f_{D_{s}}^{\tau}$ is the fraction of $\tau$ from $D_{s}(78 \%)$, from LHCb cross section measurements and LEP/B-factory branching fractions

- $\mathcal{B}\left(D_{s} \rightarrow \tau \nu_{\tau}\right)$ from arXiv:hep-ex/1201.2401

- $\epsilon$ are the efficiencies to select signal and normalisation events, calculated using MC

- Systematics cancel in ratio of efficiencies

- $\mathcal{B}\left(D_{S} \rightarrow \phi(\mu \mu) \pi\right)$ and $N_{D_{S}} \rightarrow \phi \pi$ described earlier 


\section{Peaking background: $D_{s}^{-} \rightarrow \eta\left(\mu^{+} \mu^{-} \gamma\right) \mu^{-} \bar{\nu}_{\mu}$}

- Backgrounds at LHCb from decays of heavy mesons with either:

(1) Three real final state muons

(2) One or two real muons and one or two mis-identified particles

- Most significant physical background from $D_{s}^{-} \rightarrow \eta\left(\mu^{+} \mu^{-} \gamma\right) \mu^{-} \bar{\nu}_{\mu}$

- Consider as a separate background PDF in limit calculation with a fixed normalisation

- Shape and magnitude from MC

- Total background PDF is then a combination of the above plus an additional exponential 


\section{Background fits}
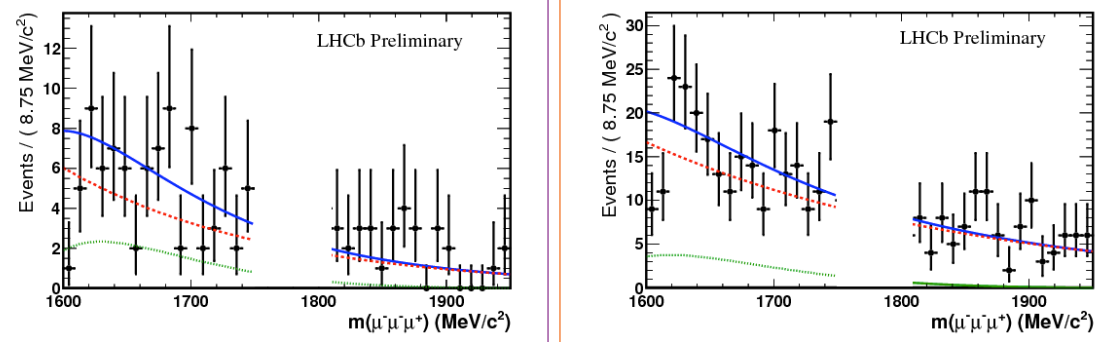

- Background estimate in signal region from data sidebands

- 4 highest (left), and second 4 highest (right) bins merged

- Combinatorial, $D_{S}^{-} \rightarrow \eta\left(\mu^{+} \mu^{-} \gamma\right) \mu^{-} \bar{\nu}_{\mu}$ and combined backgrounds

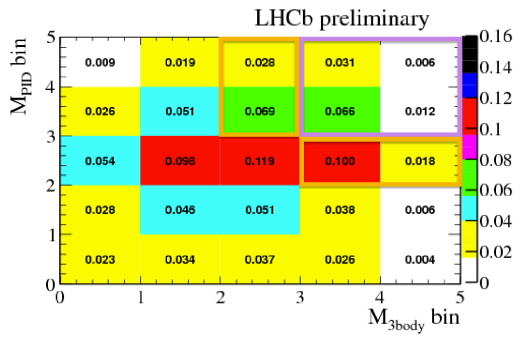




\section{Background fits}
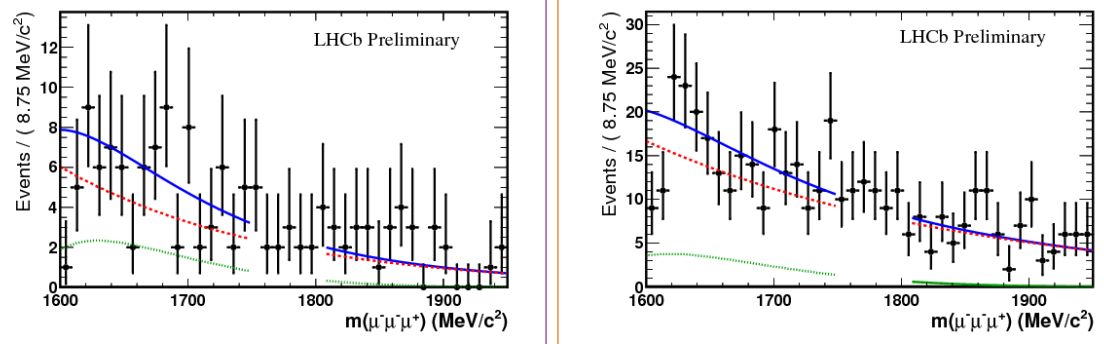

- Background estimate in signal region from data sidebands

- 4 highest (left), and second 4 highest (right) bins merged

- Combinatorial, $D_{S}^{-} \rightarrow \eta\left(\mu^{+} \mu^{-} \gamma\right) \mu^{-} \bar{\nu}_{\mu}$ and combined backgrounds

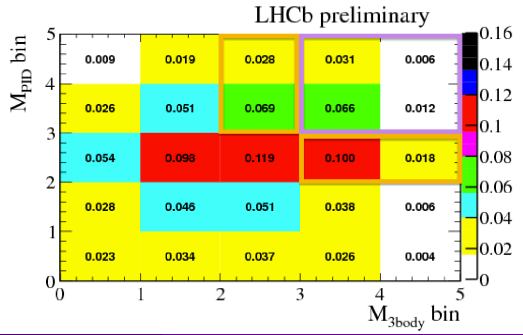




\section{Limits on $\tau^{-} \rightarrow \mu^{-} \mu^{-} \mu^{+}$}

- Limit calculated using $\mathrm{CL}_{s}$ method

- First $\tau$ LFV result at a hadron collider

- Detailed in LHCb-CONF-2012-015

- Further improvements expected in 2012

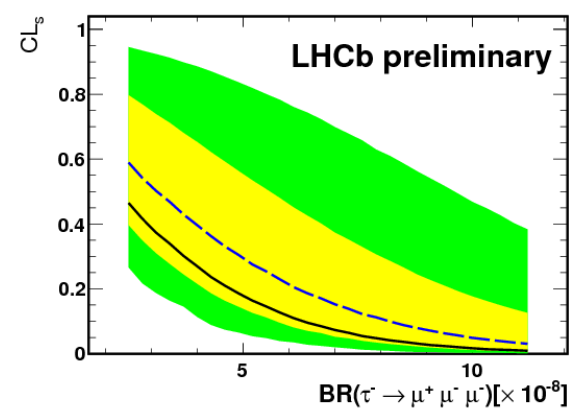

LHCb preliminary

\begin{tabular}{|c|c|c|}
\hline & Expected & Observed \\
\hline \hline 90\% C.L. & $8.2 \times 10^{-8}$ & $6.3 \times 10^{-8}$ \\
95\% C.L. & $9.9 \times 10^{-8}$ & $7.8 \times 10^{-8}$ \\
\hline
\end{tabular}


- Search for baryon number violation processes so far unsuccessful, but must have occurred in the early universe

- Conservation of angular momentum requires spin 1/2 transfer when nucleon decays to lepton: $\Delta B= \pm \Delta L$, allowing for either $\Delta(B-L)=0$ or $|\Delta(B-L)|=2$ transitions, with the former required in the $S M$ and most of its extensions

- Limits on $\tau \rightarrow \wedge h$ and $B \rightarrow \Lambda /$ in the range $10^{-7}-10^{-8}$ from $\mathrm{BaBar}^{3}$ and Belle ${ }^{4}$

- Adapt $\tau^{-} \rightarrow \mu^{-} \mu^{-} \mu^{+}$analysis to search for $\tau^{-} \rightarrow \bar{p} \mu^{+} \mu^{-}$and $\tau^{-} \rightarrow p \mu^{-} \mu^{-}$, which are both $\Delta(B-L)=0$

${ }^{3}$ Phys. Rev. 202203 D83 (2011) 091101

${ }^{4}$ arXiv:hep-ex/1207.1158 


\section{Differences between the analyses}

- Use $\mathcal{M}_{3 \text { body }}$ with $\tau^{-} \rightarrow \mu^{-} \mu^{-} \mu^{+}$tuning

- Apply hard PID cuts to reject background, optimised on signal MC and outer data sidebands

- Bin in $5 \times \mathcal{M}_{3 \text { body }}$ (top: $\tau^{-} \rightarrow \bar{p} \mu^{+} \mu^{-}$, middle: $\tau^{-} \rightarrow p \mu^{-} \mu^{-}$) and $6 \times p \mu \mu$ mass (bottom: both)

- Normalisation factor is $\sim 25 \times$ larger due to lower trigger efficiency and use of PID cuts instead of BDT

- No peaking backgrounds expected
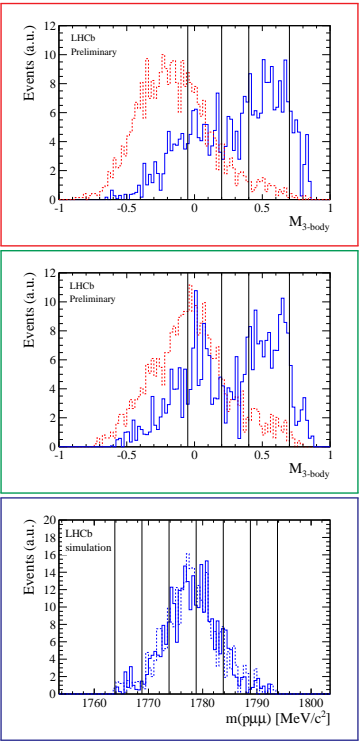


\section{Background fits for $\tau \rightarrow p \mu \mu$}

$\mathcal{M}_{\text {3body }}: 0.4-0.7$

$\mathcal{M}_{\text {3body }}: 0.7-1.0$
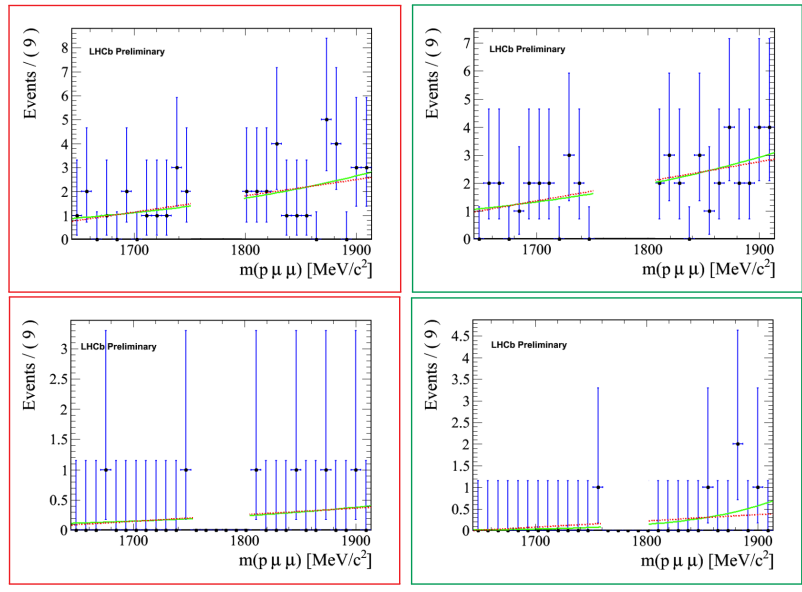

- Highest bins in $\mathcal{M}_{3 \text { body }}$ for $\tau^{-} \rightarrow \bar{p} \mu^{+} \mu^{-}$(left) and $\tau^{-} \rightarrow p \mu^{-} \mu^{-}$ (right) 


\section{Background fits for $\tau \rightarrow p \mu \mu$}

$\mathcal{M}_{\text {3body }}: 0.4-0.7$
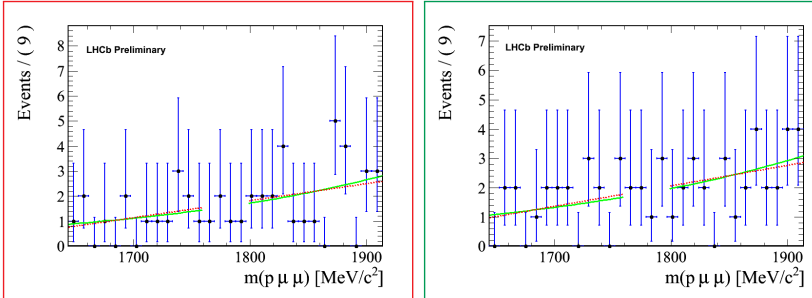

$\mathcal{M}_{\text {3body }}: 0.7-1.0$
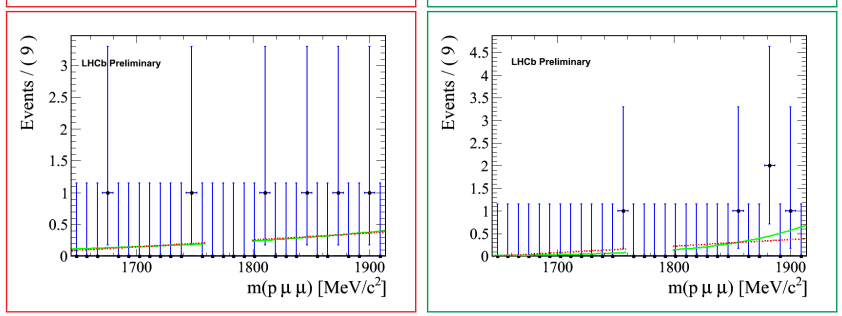

- Highest bins in $\mathcal{M}_{3 \text { body }}$ for $\tau^{-} \rightarrow \bar{p} \mu^{+} \mu^{-}$(left) and $\tau^{-} \rightarrow p \mu^{-} \mu^{-}$ (right) 


\section{Limits on}
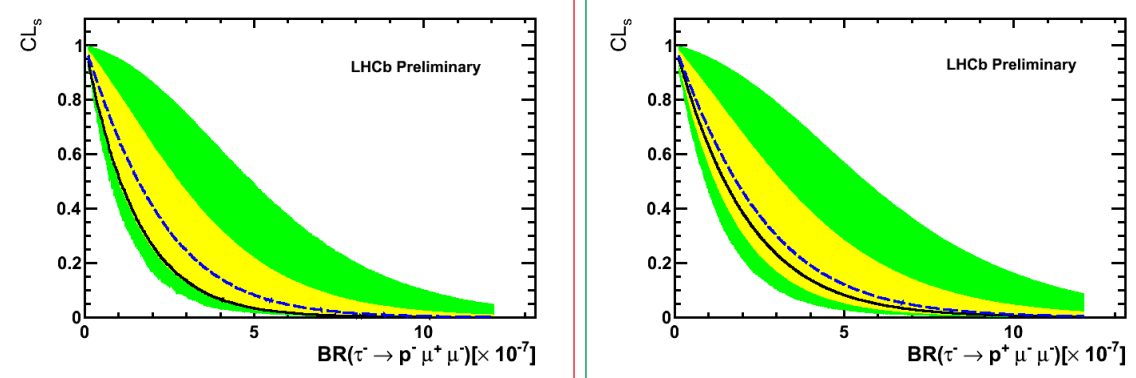

LHCb preliminary

\begin{tabular}{|l|c|c|}
\hline & Expected & Observed \\
\hline 90\% C.L. & $4.7(5.4) \times 10^{-7}$ & $3.4(4.6) \times 10^{-7}$ \\
$95 \%$ C.L. & $5.9(6.9) \times 10^{-7}$ & $4.5(6.0) \times 10^{-7}$ \\
\hline
\end{tabular}

- Detailed in LHCb-CONF-2012-027

- First limits for these decays! 


\section{Summary}

- LHCb has made the first LFV measurements at a hadron collider with $\tau^{-} \rightarrow \mu^{-} \mu^{-} \mu^{+}$

- Expect to reach/exceed B-factory limits in coming years

- First ever measurements of $\tau^{-} \rightarrow \bar{p} \mu^{+} \mu^{-}$and $\tau^{-} \rightarrow p \mu^{-} \mu^{-}$

\begin{tabular}{|c|c|c|}
\hline Channel & Expected (90\% C.L.) & Observed (90\% C.L.) \\
\hline \hline$\tau^{-} \rightarrow \mu^{-} \mu^{-} \mu^{+}$ & $8.2 \times 10^{-8}$ & $6.3 \times 10^{-8}$ \\
$\tau^{-} \rightarrow \bar{p} \mu^{+} \mu^{-}$ & $4.7 \times 10^{-7}$ & $3.4 \times 10^{-7}$ \\
$\tau^{-} \rightarrow p \mu^{-} \mu^{-}$ & $5.4 \times 10^{-7}$ & $4.6 \times 10^{-7}$ \\
\hline
\end{tabular}

- LHCb can make significant contributions to the searches for LFV and BNV 


\section{BACKUP}




\section{LHCb performance in 2012}

- Since the start of $2012 \mathrm{LHCb}$ is collecting data at $\sqrt{s}=8 \mathrm{TeV}$

- Charm cross-section is 15\% higher

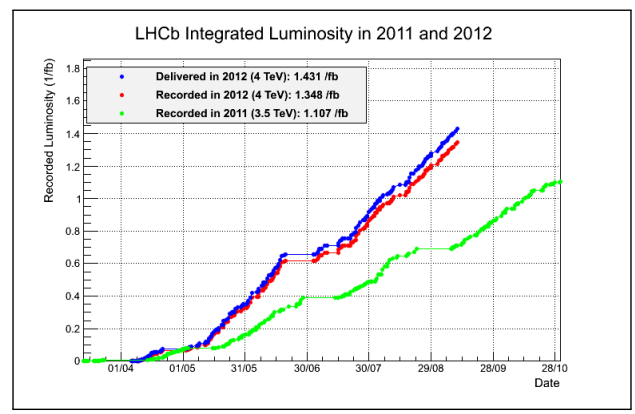

- Aiming to collect $\sim 2 \mathrm{fb}^{-1}$ before the 2013-2014 shutdown 


\section{Systematics}

- Normalisation factor contains systematic uncertainties from (in order of relative magnitude):

(1) $\tau$ production reweighting

(2) Particle ID cut efficiencies

(3) Trigger efficiency

(4) Background subtracted $D_{s} \rightarrow \phi \pi$ yield

(5) LHCb muon identification efficiency

6 Track reconstruction efficiency

(- Vertex IP resolution smearing in MC

- Difference between exponential and linear background fits included as systematic on background PDF 


\section{Observed and expected pattern of events: $\tau^{-} \rightarrow \mu^{-} \mu^{-} \mu^{+}$}

\begin{tabular}{|c|c|c|c|}
\hline $\mathcal{M}_{P I D}$ & $\overline{\mathcal{M}}_{3 \text { body }}$ & expected background & observed events \\
\hline$-1.0,-0.25$ & $-1.0,0.05$ & $24372 \pm 50$ & 23763 \\
\hline$-1.0,-0.25$ & $0.05,0.35$ & $1563 \pm 13$ & 1454 \\
\hline$-1.0,-0.25$ & $0.35,0.55$ & $407.7 \pm 6.4$ & 298 \\
\hline$-1.0,-0.25$ & $0.55,0.75$ & $144.8 \pm 3.7$ & 86 \\
\hline$-1.0,-0.25$ & $0.75,1.0$ & $12.9 \pm 1.0$ & 11 \\
\hline$-0.25,-0.125$ & $-1.0,0.05$ & $4530 \pm 22$ & 4410 \\
\hline$-0.25,-0.125$ & $0.05,0.35$ & $361.5 \pm 6.1$ & 340 \\
\hline$-0.25,-0.125$ & $0.35,0.55$ & $87.8 \pm 3.9$ & 67 \\
\hline$-0.25,-0.125$ & $0.55,0.75$ & $32.7 \pm 1.8$ & 21 \\
\hline$-0.25,-0.125$ & $0.75,1.0$ & $2.79 \pm 0.53$ & 3 \\
\hline$-0.125,-0.025$ & $-1.0,0.05$ & $2103 \pm 15$ & 2061 \\
\hline$-0.125,-0.025$ & $0.05,0.35$ & $209.0 \pm 4.8$ & 194 \\
\hline$-0.125,-0.025$ & $0.35,0.55$ & $56.8 \pm 2.4$ & 54 \\
\hline$-0.125,-0.025$ & $0.55,0.75$ & $19.8 \pm 1.2$ & 29 \\
\hline$-0.125,-0.025$ & $0.75,1.0$ & $3.28 \pm 0.58$ & 1 \\
\hline$-0.025,0.05$ & $-1.0,0.05$ & $463.7 \pm 6.9$ & 460 \\
\hline$-0.025,0.05$ & $0.05,0.35$ & $49.1 \pm 2.2$ & 43 \\
\hline$-0.025,0.05$ & $0.35,0.55$ & $15.4 \pm 1.2$ & 13 \\
\hline$-0.025,0.05$ & $0.55,0.75$ & $7.10 \pm 0.82$ & 3 \\
\hline$-0.025,0.05$ & $0.75,1.0$ & $0.46 \pm 0.17$ & 1 \\
\hline $0.05,1.0$ & $-1.0,0.05$ & $106.2 \pm 3.3$ & 107 \\
\hline $0.05,1.0$ & $0.05,0.35$ & $8.59 \pm 1.8$ & 11 \\
\hline $0.05,1.0$ & $0.35,0.55$ & $5.85 \pm 0.74$ & 5 \\
\hline $0.05,1.0$ & $0.55,0.75$ & $2.12 \pm 0.40$ & 4 \\
\hline $0.05,1.0$ & $0.75,1.0$ & $0.49 \pm 0.20$ & 0 \\
\hline
\end{tabular}

- Lowest likelihood bins found to have no effect on the limit 


\section{Model dependence: $\tau^{-} \rightarrow \mu^{-} \mu^{-} \mu^{+}$}

- Branching fraction depends on new physics model which is assumed

- MC samples generated assuming a flat phase space distribution

- Combined selection \& trigger efficiency found to remain constant within $15 \%$ across the whole dimuon mass spectrum

- BDT response is also flat as a function of dimuon mass 


\section{Observed and expected pattern of events: $\tau \rightarrow p \mu \mu$}

\begin{tabular}{|c|c||c|}
\hline $\mathcal{M}_{3 \text { body }}$ & Expected background & Observed events \\
\hline$-1.00,-0.05$ & $85.2 \pm 3.3$ & 91 \\
\hline$-0.05,0.20$ & $29.5 \pm 2.0$ & 32 \\
\hline $0.20,0.40$ & $9.8 \pm 1.1$ & 5 \\
\hline $0.40,0.70$ & $5.48 \pm 0.85$ & 4 \\
\hline $0.70,1.00$ & $0.78 \pm 0.32$ & 0 \\
\hline
\end{tabular}

\begin{tabular}{|c|c||c|}
\hline $\mathcal{M}_{3 \text { body }}$ & Expected background & Observed events \\
\hline$-1.00,-0.05$ & $47.4 \pm 2.5$ & 69 \\
\hline$-0.05,0.20$ & $32.0 \pm 2.0$ & 33 \\
\hline $0.20,0.40$ & $9.0 \pm 1.1$ & 7 \\
\hline $0.40,0.70$ & $6.1 \pm 0.90$ & 4 \\
\hline $0.70,1.00$ & $0.65 \pm 0.11$ & 0 \\
\hline
\end{tabular}

- Lowest likelihood bin found to have no effect on the limit 


\section{Differences between}

\section{and}

- $\tau^{-} \rightarrow \bar{p} \mu^{+} \mu^{-}$and $\tau^{-} \rightarrow p \mu^{-} \mu^{-}$can probe different NP models:

\begin{tabular}{|c|c|c|c|}
\hline Channel & $\Delta(\tau)$ & $\Delta(\mu)$ & $\Delta$ (baryon) \\
\hline$\tau^{-} \rightarrow \bar{p} \mu^{+} \mu^{-}$ & -1 & 0 & -1 \\
\hline$\tau^{-} \rightarrow p \mu^{-} \mu^{-}$ & -1 & +2 & +1 \\
\hline
\end{tabular}

- $\tau^{-} \rightarrow \bar{p} \mu^{+} \mu^{-}$similar to channels studied at BaBar and Belle

- Differences in upper limits due to:

(1) LHCb trigger

2 Detector acceptance to pairs of tracks of opposite or same charge $\rightarrow \tau^{-} \rightarrow \bar{p} \mu^{+} \mu^{-}$has slightly better mass resolution

(3) Nature of combinatorial background 